\title{
DIFFUSE REFLECTANCE SPECTRA OF COALS IN THE UV-VISIBLE AND NEAR- IR REGIONS
}

\author{
Manoj B.* and Kunjomana A.G.**
}

\begin{abstract}
Chemical leaching of sub-bituminous coal with inorganic and organic leachants has been monitored by the UV-Visible and Near-IR spectra with the diffuse reflectance method. The shape and infensity of the observed absorption bands changed with leaching, reflecting the solubilization of the aromatic molecules and the degree of the intermolecular interaction between organic and inorganic phases. The absorption maximum of benzene-oxygen system was found between 235-270 $\mathrm{nm}$ and is showing a red shift with leaching. The characteristic naphthalene ring systems $(220 \& 280 \mathrm{~nm})$ are masked by the absorption regions of monoaromatic rings; indicating the content of napthalenoid hydrocarbon is very low. The bands observed in the visible region $(450 \mathrm{~nm})$ is attributed to $\mathrm{SO}_{2}$ in the sample and with leaching this band is showing a red shift. The weak band of the $680 \mathrm{~nm}$ is attributed to the $\Pi$ I- $\Pi^{*}$ electronic transitions of the polynuclear aromatic hydrocarbons which shows red shift with leaching.
\end{abstract}

Keywords: diffuse reflectance spectrum; leaching; solubilization.

* Corresponding author, Department of Physics, Christ University, Bangalore-560 029, manoj.b@Christuniversity.in

** Department of Physics, Christ University, Bangalore-560 029. 


\section{Introduction}

Over the past 15 years, diffuse reflectance spectrometry has been applied to the mid-IR spectra of coal and carbons; this method gives very sharp and reliable IR bands. Osamu lto et al $(1992,1993)$ reported that the broad absorption bands observed in the region between $4000-6000 \mathrm{~cm}^{-1}$ by this technique are attributable to electronic transitions and not to the effect of scattering. For the UV-visible and near - IR regions above $6000 \mathrm{~cm}^{-1}$, however, diffuse reflectance spectrometry characterization of coal samples are scarce, even though this method has widely applied to solid organic and inorganic compounds. The UV-VIS-NIR spectra give valuable information about the structure of the molecule because the absorption of UV and visible light involves promotion of electron in $\sigma$ and $\Pi$ orbitals from ground state to higher energy state. In this paper, it is reported that the absorption spectra in the UV-Visible and near regions, as measured by diffuse reflectance, change with coal rank and the chemical leaching, and time of leaching.

\section{Materials and methods}

Sub-bituminous coal sample from Godavari coal fields are brought to the lab as it is and kept in open for 3 months. The sample is well powdered and treated with EDTA for $24 \mathrm{hrs}$ at $27^{\circ} \mathrm{C}$. The precipitate is removed and residue is washed with water and filtered. The sample is treated with acetic acid for period of 1 day, 2 days and 3 days (GEAI-GEA3). After the specified contact time sample is washed with distilled water, filtered and dried at a temperature of $80^{\circ} \mathrm{C}$ and allowed to cool slowly in a dessicator. Virgin sample is treated with dilute nitric acid for $24 \mathrm{hrs}$ and followed by concentrated HF of concentration $40 \%, 30 \%, 20 \%$ and $10 \%$ for an hour (GNF1-GNF4). The residue is separated and dried as discussed above.

Diffuse reflectance a spectrum of coals in the UV-Visible and near-IR regions is carried out using a Cary 500 spectrometer equipped with a Praying Mantis diffuse reflectance accessory DRA.

\section{Result and Discussion}

The electronic absorption spectra of sub-bituminous and bituminous coal are measured by the diffuse reflectance spectrometry in the UV-Visible and near $I R$ regions (200-800 nm). In the measured spectra (Figure 1 and Figure 2) the $\sigma$ band $(260-270 \mathrm{~nm})$ has defined structure. This absorption increases with leaching with strong absorption for HF leached samples. The general shape of the spectrum is characteristic for hydrocarbons with a single benzene ring. The presence of 
napthalenoid hydrocarbons will show up in the spectrum and can be judged qualitatively only in the $320 \mathrm{~nm}$ regions, where benzoid hydrocarbon give practically no absorption of UV radiation. The two principal bands that are characteristic for the naphthalene system $(220 \& 280 \mathrm{~nm})$ are masked by the absorption regions of monoaromatic rings; this indicates that the content of napthalenoid hydrocarbon is very low (Siryuk et al 1979, Satoshi Hashimoto 1989). The absorption maximum of benezene-oxygen (1/1) system was found between $235-270 \mathrm{~nm}$. This is due to the benezene-oxygen charge transfer band and is showing a red shift with leaching, which is more prominent with $\mathrm{HF}$ leaching $(20 \%$ and $40 \%)$ and pretreated acetic acid sample (GEA3).

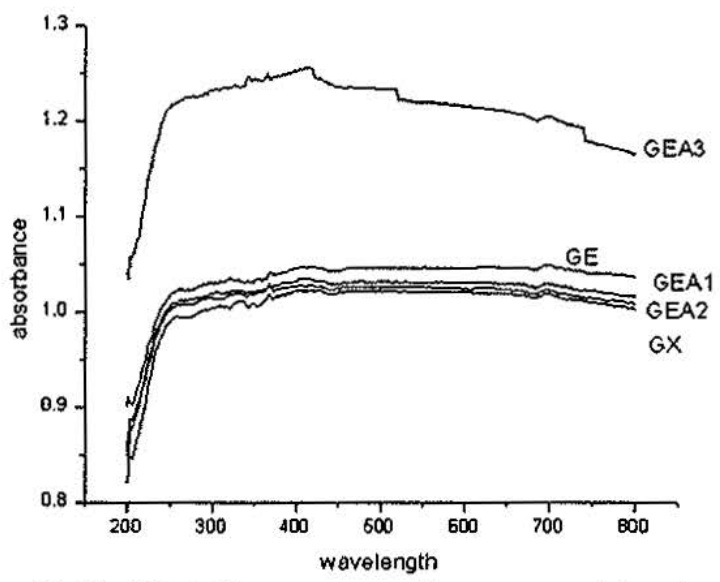

Figure 1 - UV-Visible-NIR spectrum of acetic acid leached sample

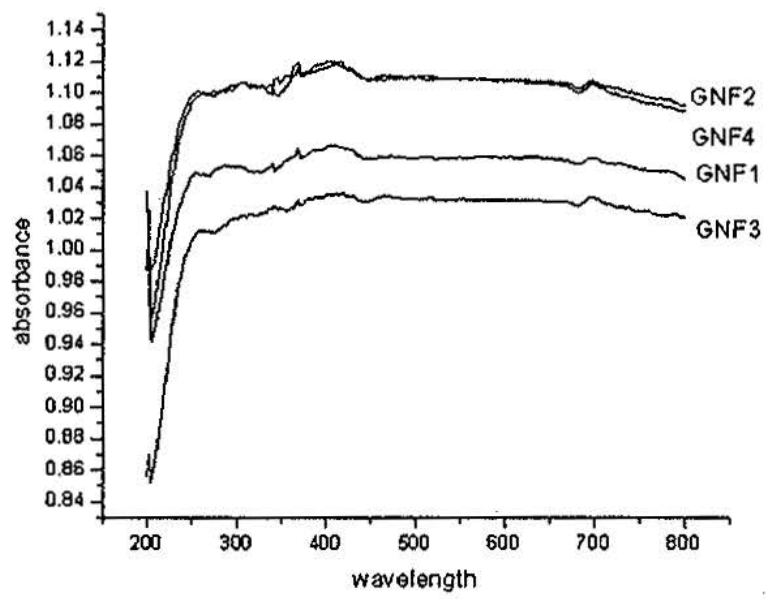

Figure 2 - UV-Visible -NIR spectrum of HF leached sample 
Olaijire et al (2007) reported prominent peaks at $400 \mathrm{~nm}$ (Soret band) and at wavelength ranges of $535-550 \mathrm{~nm}$ ( $\beta$ band) and $565-600 \mathrm{~nm}$ ( $\alpha$ band) for the Nigerian coal minerals. In present study, sample shows peaks at $400 \mathrm{~nm}$ which increase with organic acid leaching and is maximum for EDTA and acetic acid leaching. At 409, 450,503, $518 \mathrm{~nm}$ there is a correlation between volatile matter and diffuse reflectivity. Frederick $J$ et al (1983) reported two maxima at 620 and $577 \mathrm{~nm}$ in New Mexican coals. They designated the most intense band at $620 \mathrm{~nm}$ to the color of vitrinite in transmission. The absorption of the particular maxima increases with Organic acid leaching as well as HF leaching with maximum absorption for sample treated with acetic acid and EDTA for three days (GEA3).

The sample is almost transparent in the region $475 \mathrm{~nm}$ to $650 \mathrm{~nm}$ and near IR region. There are small distinct absorption peaks in the $U V$ region and $680 \mathrm{~nm}$. The intensity of the broad absorption in the visible $(680 \mathrm{~nm})$ and near $\mathbb{R}$ region $(759 \mathrm{~nm})$ are attributable to the $\Pi-\Pi^{*}$ electronic transitions of the poly nuclear aromatic hydrocarbons, increases with the rank of coal (Osamu lto 1992). The intensity of this band increases with chemical leaching is an indication of electronic transitions with leaching.

There are weak bands observed in the visible region $(450 \mathrm{~nm})$ is due to the presence of $\mathrm{SO}_{2}$ in the sample. This band is showing a strong red shift with $\mathrm{HF}$ leaching as well as acetic acid leaching. Red shift is observed for pretreated sample leached with acetic acid for 3 days. Leaching increases electron releasing group of the sample which in turn increases both the wavelength and intensity of the secondary absorption band.

\section{Conclusion}

Chemical leaching processes of sub-bituminous coal have been monitored by the UV-Visible and near IR spectra measured with diffuse reflectance method. The shape and intensity of the observed absorption bands changed with leaching of the sample, reflecting the degree of the intermolecular interaction. In the measured spectra, the o band (260-270 nm) has defined structure. This absorption increases with leaching and is highest for GAE sample than GNF sample. This part of the spectrum is characteristic for hydrocarbons with single benzene ring. The characteristic bands of naphthalene ring system $(220$ and $280 \mathrm{~nm})$ are masked by the absorption regions of the monoaromatic rings; indicating the content of napthalenoid is very low. The band in the region $680 \mathrm{~nm}$ is attributed to the $\Pi-\Pi^{*}$ electronic transitions of the polynuclear aromatic hydrocarbons. Intensity of this band increases with chemical leaching is an indication of increase of electronic transitions with leaching. The weak bands observed in the visible region $(450 \mathrm{~nm})$ are due to the presence of 
$\mathrm{SO}_{2}$ in the sample and is showing a red shift with $\mathrm{HF}$ and acetic acid leaching. The electron releasing groups increases both the wavelength and intensity of the secondary absorption band.

\section{Acknowledgments}

The authors would like to thank the research and development centre, Bharthiar University for allowing registering as a research scholar. Thanks are due to Christ University for providing financial assistance to carry out research.

\section{References}

1. Osamu Ito, "Diffuse reflectance spectra of coals in the UV-Visible and Near-IR regions" 1992, Energy and Fuels, 6, 662-665.

2. Osamu Ito "UV-Visible and near-IR spectra of heat-treated pitches during mesophase formation", 1993, Carbon, 31(3), 401-406.

3. A.G.Siryuk, F.D. Radchenko and M.M. Fernandes-Games, "Analysis of aromatic hydrocarbons in fuels by means of UV absorption spectra", 1979, Chemistry and Tech. Of Fuels and oils, 15 (7), Doi: 10.10007/BF00723711,.530-534.

4. Satoshi Hashimoto and Hajime Akimoto, "UV absorption spectra and photochemical reactions of simple aromatic hydrocarbons in the Cryogenic oxygen matrix", 1989, J.Phys.Chem, 93, 571-577.

5. A.A.Olaijire, A.B.Ameen, M.Abdlil-Hammed and F.A. Adekola. "Occurrence and distribution of metals and porphyrins in nigerial coal minerals",2007, Journal of Fuel chemistry and technology, 35(6), 641-647.

6. Frederick J.Kuellmes, Frank Kimbler and Janet Nuter, "Preliminary report on the diffuse reflectivity of some New Mexico coals", 1983, International Journal of Coal geology, 2(3), 261.277. 